Kontakt:

harald.spirig@aon.at

schmied@euro.centre.org

irene.berlach-pobitzer@univie.ac.at

\section{Fußnoten:}

1 Spirig, Harald: Die >Drogenfreie Zone in der Justizanstalt Hirtenberg. Wien, 2000.

2 Spirig, Harald; Ess-Dietz, Octavia: Gesundheitspräventive Maßnahmen im Österreichischen Strafvollzug. European Network on HIV/AIDS and Hepatitis Prevention in Prisons, 2001
3 Dieses Gefängnis ist in Größe und Art der Gefangenenpopulation mit der JA Hirtenberg am ehesten vergleichbar, verfügt aber über keine Drogenfreie Zone.

4 s. auch: Spirig, Harald: Die >Drogenfreie Zone $<$ in der Justizanstalt Hirtenberg. Wien, 2000

5 Die addierte Haftdauer nach Wiederverurteilungen etwa ist bei Entlassenen aus der DFZ signifikant geringer: Eta $2=0,22 ; 22 \%$ der Varianz werden durch die drei Gruppen erklärt.

6 Jede Vorstrafe $=1$ Punkt Je 6 Monate Haftzeit (Vorstrafen bis zur Entlassung) $=1$ Punkt.
Unterschiedliche Delikte wurden mit unterschiedlichen Punktzahlen versehen: Eigentumsdelikt $=1$ Punkt; Körperverletzung $=2$ Punkte; Raub = 2 Punkte; Suchtgiftmissbrauch = 1 Punkt; Suchtgifthandel $=2$ Punkte

7 Gruppe 1: nicht signifikant, DFZ 0,64, Hirtenberg-Normalvollzug 0,92 , JA Suben 0,74

Gruppe 2: signifikant, DFZ 0,49, Hirtenberg-Normalvollzug 1,68, JA Suben 0,93, Eta2= 0,098; Knapp 10\% der Varianz werden durch die drei Gruppen erklärt. Gruppe 3: signifikant, DFZ 0,81, Hirtenberg-Normalvollzug 2,03,
JA Suben 1,24, Eta2 $=0,062,6,2 \%$ der Varianz werden durch die drei Gruppen erklärt.

Gruppe 4: signifikant, DFZ 1,16, Hirtenberg-Normalvollzug 1,30 , JA Suben 2,00, Eta2 $=0,053,5,3 \%$ der Varianz werden durch die drei Gruppen erklärt.

8 Kursiv gestellte Texte sind wörtliche Zitate aus den Interviews.

9 Zur Unterscheidung von output und outcome s. den Beitrag von Bernd Maelicke: Rationalität der Justiz in Neue Kriminalpolitik 2/2004

10 Spirig, Harald: Die , Drogenfreie Zone in der Justizanstalt Hirtenberg. Wien, 2000 .

\title{
Lebenslange Verwahrung angeblich nicht therapier- barer und extrem gefährlicher Sexualstraftäter seit 1998 in Deutschland und der Schweiz
}

Monika Frommel

\begin{abstract}
Am 29.Juli 2004 trat das neue Bundesgesetz, das künftig anstelle der nichtigen Landesgesetze die nachträgliche Sicherungsverwahrung in § 66 b StGB regelt, in Kraft. Auch die Schweiz wird demnächst die durch Art 126 a der Schweizer Verfassung mögliche und ggf. lebenslange Verwahrung im einzelnen in einem neu gefassten AT des Schweizer StGB detailliert regeln ${ }^{1}$. Damit haben sich die Hoffnungen von Frieder Dünkel ${ }^{2}$ zerschlagen, die Grünen könnten den Entwurf der Bundesregierung noch entscheidend verändern. Es bleibt somit bei dem von ihm beschriebenen Pyrrhussieg der Gegner der nachträglichen Sicherungsverwahrung durch Landesgesetze.
\end{abstract}

$\int$ ie Reform des Sexualstrafrechts ist leider seit den 1980er Jahren immer zu eng verknüpft mit Debatten um Mindeststrafen. In der Zeit der wachsenden feministischen Empörung über die damals bagatellisierenden Strategien im Umgang mit diesem Problem war es verständlich, dass eine intensivere Strafverfolgung mit "mehr Strafe« assoziiert war, aber mittlerweile sind auch dies Pyrrhussiege. Zwar ging es immer auch um eine kluge Politik: primäre Prävention durch Antidiskriminierung und Antidiskriminierung durch Strafrechtsreform, eine Politik also, die ein spezifisches Risiko vorwiegend weiblicher und kindlicher Opfer insgesamt gesehen eher pragmatisch anging und die aus meiner Sicht auch langfristig erfolgreich war und die auch künftig noch erfolgreich sein wird. Aber neben dieser aufgeklärten Debatte gab es immer zugleich auch zwei weitere, in meinen Augen atavistische Kampagnen, eine sich radikalfeministische gebende Strafverschärfungsdebatte3 und eine gegen diese Sicht gerichtete Position, die den »Feind « nicht in Traditionen der Geschlechterhierarchie verortet, sondern den Täter als Person »bekämpft«. Schließlich hat die vermehrte Einschaltung von psychiatrischen Gutachtern und die Einbindung sog. Fachkommissionen, etwa in der Schweiz, Typologien des dissozialen Kriminellen gefördert. Medial werden daraus unverbesserliche Vergewaltigers und Kinderschänder, so dass sich ein pseudowissenschaftliches Konstrukt bildet und die aktuelle Politik prägt. Aber bisweilen meinen auch Psychiater, ihre auf Konventionen aufgebauten Prognoseverfahren seien mehr als tautologische Feststellungen. Denn wer hätte je bezweifelt dass eine hohe und intensive Delinquenzgeschichte ungünstig ist für ein zu prognostizierendes künftiges Verhalten4. Habituelle Kriminalität wird danach definiert als einge- schliffenes Verhaltensmuster, in der Biografie erkennbar mit einem Delinquenzbeginn schon in Kindheit oder Jugend, Herkunft aus dissozialem Milieu, grausamen Taten in der Vorgeschichte und gehäuftem Lockerungs- oder Bewährungsversagen. Rein praktisch ist es kaum zu bezweifeln, dass die Delinquenzgeschichte ein statistisch hochsignifikater Prädiktor ist, aber die Trivialität dieser Aussage sollte auch skeptisch machen, ebenso der historisch geschulte Blick. Wir haben es in der Tat mit einer Wiederbelebung des schon 1882 von Franz von Liszt etwa so beschriebenen »gefährlichen Gewohnheitsverbrecher« zu tun.

Polarisierungen provozieren Befürworter und Gegner. Die einen wünschen, die anderen bekämpfen eine Sicherheitspolitik, von der sie nicht sagen, ob sie tatsächlich mehr Sicherheit bringt bzw. ob sie tatsächlich prozessuale Rechte untergräbt. Nicht minder schlicht war und ist die Verarbeitung dieser Positionen innerhalb des Politikbetriebs. In Deutschland ist dieser zunehmend anfällig (nicht ganz aber doch relativ unabhängig vom jeweiligen Personal) für Alibigesetze. In der Schweiz kommt noch der Populismus jederzeit möglicher Volksbegehren hinzu. Beide Rechtskulturen tendieren aber zur Zeit dazu, das Problem zu dramatisieren und Scheinlösungen zu präsentieren.

Die deutsche Gesetzgebung betreibt seit 1998 eine Politik der permanenten Reform der Sicherungsverwahrung (1998 eine erweiterte, 2002 eine vorbehaltene und seit 2004 eine nachträgliche ${ }^{5}$ ). Die Gründe sind durchsichtig, weil der oder die LandesjustizministerIn bei jeder Rückfalltat darlegen muss, dass es sich um einen Vollverbüßer gehandelt habe und man nun einmal durch das geltende Recht gebunden sei. Kein Wunder, dass dann diejenigen, die politisch auf 
"Sicherheitspolitik« (ohne zu sehen, wie riskant eine solch kurzatmige Politik ist) festgelegt sind, das jeweils geltende Recht permanent der jeweils aktuellen Aufregung anpassen wollen.

Im deutschsprachigen Rechtskreis gilt seit Beginn des 20. Jh. das von Stooß entwickelte Prinzip der Zweispurigkeit. Maßregeln sind danach etwas ganz anderes als Strafen, ein Gedanke, der explizit in $\S 2$ Abs. 6 des deutschen StGB zum Ausdruck kommt und zur Folge hat, dass dort Maßregeln vom Rückwirkungsverbot ausgenom- auch der schweizer Neuregelungen eher abzulehnen sein. Das BVerfG hatte im Februar 2004 diese Frage lediglich deshalb nicht entschieden, weil es an das GG und die dort vorausgesetzte Zweispurigkeit gebunden und Landesgesetze $\mathrm{zu}$ beurteilen hatte, die ohnehin nach einer gewissen Frist - für nichtig erklärt werden mussten, da sie nicht einmal von einem kompetenten Gesetzgeber erlassen worden waren. Zwar sieht das zweispurige Modell die begriffliche Unterscheidung zwischen Prävention und Repression i.S. einer Vergeltung des zurechenbar verwirklich-

\section{In Deutschland fehlt ein Konzept für die große Gruppe der gestörten, aber nicht eindeutig schuldunfähigen bzw. vermindert schuldfähigen Sexualstraftäter und es fehlt ein ambulantes Netz der Sicherung.}

men werden. Eine vorbeugende Verwahrung wird danach behandelt wie ein Eingriff in Freiheitsrechte im überwiegenden Allgemeininteresse. Man muss zum Zeitpunkt des Eingriffs, aber nicht schon zum Zeitpunkt der Anlasstat eine Rechtsgrundlage für die Verwahrung haben. Deshalb setzen alle Populisten auf die nachträgliche Sicherungsverwahrung. Aber von dieser terminologischen Unterscheidung wird sich der Strassburger Gerichtshof bei einer Individualbeschwerde (eine abstrakte Normenkontrolle ist nicht vorgesehen) nur wenig beeindrucken lassen. Die neuere Strassburger Rechtsprechung lässt zwar eine unbefristete Verwahrung $\mathrm{zu}$, aber nur wenn sie vom erkennenden Gericht im Strafurteil vorbehalten worden ist. Aber genau dies will weder das Schweizer Volksbegehren noch der deutsche Gesetzgeber mit dem neuen $\S 66$ b StGB. Man möchte nicht auf die Tat, sondern auf die persönliche Entwicklung des Verurteilten im Vollzug reagieren, der Sache nach also das frühere Urteil korrigieren, was erkennbar Probleme aufwirft, da der Grundsatz des Verbots der Doppelbestrafung eindeutig berührt ist. So gesehen dürfte die EMRK-Konformität sowohl der deutschen als rechtliche Gehör. Konzipiert ist diese Intervention also zur Zeit nach rein präventiven Gesichtspunkten. Aber trotz dieser erheblichen Zugeständnisse in der Vergangenheit blieb man in der Schweiz dennoch nicht von noch weitergehendem Populismus verschont. Auch der bereits beschlossene moderate Entwurf zur Sicherungsverwahrung nach Art. 64 SchwStGB, der eine Verwahrung auf unbestimmte Zeit ermöglicht, verhinderte nicht, dass es nun noch einen zweiten Weg in die Verwahrung gibt. Dass die Initiative nicht mit der EMRK kompatibel ist, ändert nämlich zunächst nichts an ihrer Wirksamkeit. Überlegt wird daher, wie mit dem neuen Art. 123 a der Schweizer Verfassung zu verfahren ist. Soll man abwarten, ob es zu einer Individualbeschwerde zum Europäischen Gerichtshof für Menschenrechte kommt oder soll der einfache Gesetzgeber trotz der Unvereinbarkeit mit der EMRK "nachbessern " und das sperrige Anliegen des Volkes wenigstens in eine technisch handhabbare Form bringen? Stefan Trechsel ist sich darüber im Klaren, dass sein im Folgenden abgedruckter Vorschlag ein Versuchsballon ist. Aber als unverbindlicher Einstieg in die nun unumgängliche Schweizer Debatte sei er hier kurz dargestellt: (S. 88)

Es ist kein Geheimnis, dass weder in Deutschland noch in der Schweiz die sachverständige Literatur derartigen Forderungen zustimmt. Schon 1998 zum Gesetz zur Bekämpfung von Sexualdelikten und anderen gefährlichen Straftätern vom 26.01.1998 prophezeiten erfahrende deutsche Praktiker, was nun auch eingetreten ist: eine Überfüllung der Haftanstalten und eine Überbelegung des Maßregelvollzugs in den psychiatrischen Krankenhäusern. Auch die einhellige Ablehnung der nun für den Bund hastig formulierten und ebenso übereilt beschlossene nachträgliche Sicherungsverwahrung durch diejenigen, die damit arbeiten müssen, wird bestätigen, wie sinnwidrig es ist, die knappe Ressource der mittlerweile fachlich gut weiter gebildeten Gutachter viel zu spät, nämlich erst vor der Entlassung und in erster Linie nur im Rahmen der zu überprüfenden Vollzugslockerung einzubinden. Aber der gemeinsame Protest unterschiedlicher akademischer Berufsgruppen hat lediglich dazu geführt, den Zusammenhang zwischen Wissenschaft und Kriminalpolitik insgesamt zu lockern, um den scharfen Blick skeptischer Beobachter los zu werden.

Was tun? Soll man die Schwächen deutlich in den Vordergrund stellen, nach dem Motto, nur wenn alles erst noch viel schlimmer wird, kann sich das Blatt zum Bessern wenden, oder versuchen, den Schaden möglichst gering zu halten, zu retten, was zu retten ist. Antizipiert wird also kein Verfahren vor dem Europäischen Gerichtshof in Strassburg, weder gegen die Schweizer Initiative noch gegen das künftige deutsche Gesetz,

noch ausgehen werden.

Rechtstaatliche Bedenken kümmerten in letzter Zeit weder das Schweizer Volk noch die deutsche Politik. Die Schweizer Politik war zwar so umsichtig und bildete (unter dem Vorsitz von Volker Dittmann, Forensische Abteilung der psychiatrischen Universitätsklinik Basel) eine professionell arbeitende Prognosekommission, die frühzeitig die Einweisung in die jeweils passende Einrichtung prüft und regelmäßig korrigiert. Der Grundgedanke ist bestechend, aber die rechtliche Rahmung für die Arbeitsweise dieser Kommission ist aus rechtsstaatlicher Perspektive umstritten. In einer sehr detaillierten Analyse zeigt Günter Stratenwerth ${ }^{6}$ die Schwachstellen des $\mathrm{zu}$ erwartenden neuen Schweizer Rechts. Kritisiert wird fehlende Transparenz und das bei einer Freiheitsentziehung unzureichend ausgestaltete

\section{Art 123 a der Schweizer Verfassung:}

1 Wird ein Sexual- oder Gewaltstraftäter in den Gutachten, die für das Gerichtsurteil nötig sind, als extrem gefährlich erachtet und nicht therapierbar eingestuft, so ist er wegen des hohen Rückfallrisikos bis an sein Lebensende zu verwahren. Frühzeitige Entlassung und Hafturlaub sind ausgeschlossen.

2 Nur wenn durch neue, wissenschaftliche Erkenntnisse erwiesen wird, dass der Täter geheilt werden kann und somit keine Gefahr mehr für die Öffentlichkeit darstellt, können neue Gutachten erstellt werden. Sollte aufgrund dieser neuen Gutachten die Verwahrung aufgehoben werden, so muss die Haftung für einen Rückfall des Täters von der Behörde übernommen werden, die die Verwahrung aufgehoben hat.

3 Alle Gutachten zur Beurteilung der Sexual- und Gewaltstraftäter sind von mindestens zwei voneinander unabhängigen, erfahrenen Fachleuten unter Berücksichtigung aller für die Beurteilung wichtigen Grundlagen zu erstellen. 


\section{Vorschlag von Stefan Trechsel}

Einzufügen ist nach Art. 64 SchwStGB (Sicherungsverwahrung auf unbestimmte Zeit) ein neuer Art. 65a SchwStGB: Lebenslange Verwahrung, Voraussetzungen und Vollzug

1 Das Gericht ordnet die lebenslange Verwahrung an, wenn der Täter aus sexuellen Beweggründen vorsätzliche Tötung, Art. 111, Mord, Art. 112, oder schwere Körperverletzung, Art. 122, sexuelle Nötigung oder Vergewaltigung an einem Kind, Art. 187 in Verbindung mit Art. 189 oder 190, qualifizierte sexuelle Nötigung, Art. 189 Abs. 3, oder qualifizierte Vergewaltigung, Art. 190 Abs. 3, begangen und mindestens eine weitere Tat der gleichen Art verübt oder versucht hat, wenn eine sehr hohe Wahrscheinlichkeit besteht, dass er weitere Taten der gleichen Art und Schwere begehen wird und wenn es nicht möglich ist, diese Gefahr durch eine Behandlung herabzusetzen.

2 Der Vollzug der Freiheitsstrafe geht der lebenslangen Verwahrung voraus.

3 Die Verwahrung wird in einer Massnahmevollzugseinrichtung oder in einer Strafanstalt nach Artikel 76 Absatz 2 vollzogen. Die öffentliche Sicherheit ist zu gewährleisten. Es wird kein Ausgang oder Hafturlaub gewährt. Der Täter ist psychiatrisch oder psychologisch zu betreuen, sofern dies zweckmässig ist.

\section{Art. $65 b$ Dauer der lebenslangen Verwahrung}

1 Lassen neue wissenschaftliche Erkenntnisse erwarten, dass eine Behandlung des Verwahrten seine Gefährlichkeit herabsetzt, ist sie ihm anzubieten. Unterzieht er sich der Behandlung und zeitigt sie erste Erfolge, beendet das Gericht, das sie angeordnet hat, die lebenslange Verwahrung.

2 An Stelle der lebenslangen Verwahrung tritt eine Massnahme gemäss Artikel 59 oder 64 (Maßregelvollzug oder Sicherungsverwahrung).

3 Die bedingte Entlassung richtet sich nach den Vorschriften, die für die angeordnete Massnahme gelten.

Art. $65 c$ Verfahren

1 Mit dem Urteil entscheidet das Gericht gestützt auf die Gutachten zweier Sachverständiger vorläufig über die Anordnung der lebenslangen Verwahrung.

2 Auf das Ende der unverkürzten Strafverbüssung hin entscheidet das Gericht, welches vorläufig die lebenslange Verwahrung angeordnet hat, gestützt auf neue Gutachten von zwei Sachverständigen endgültig.

3 Wird der Verwahrte gemäss Artikel 65b Absatz 1 behandelt, entscheide das Gericht von Amtes wegen oder auf Antrag des Verwahrten oder der Anstaltsleitung, ob erste Erfolge eingetreten sind. Ist dies der Fall, beendet es die lebenslange Verwahrung und ordnet eine Massnahme gemäss Artikel 59 oder 64 an (Maßregelvollzug oder Sicherungsverwahrung).

sondern es wird konform zu diesen Initiativen versucht das schlimmste zu verhindern:

Im Gegenzug versuche ich zu antizipieren, wie Vollstreckungsgerichte in Deutschland mit dem nicht minder rätselhaften neuen Bundesgesetz umgehen könnten; denn abzuwarten, bis erneut dessen Verfassungs - oder EMRK-Konfor- zen umwechseln. Versuchen wir einmal einen Ansatz $\mathrm{zu}$ einer folgenreichen Präzisierung:

Abgestellt wird in $\S 66$ b n.F. StGB in beiden Absätzen auf "neue Tatsachen", die erst "nach der Verurteilung « die (in $\S 66$ b weiter und weniger einschränkend umschriebene) besondere Gefährlichkeit erkennen lassen (in $\$ \S 66,66$ a muss die Gefährlichkeit auf einem »Hang zu erheblichen Straftaten « beruhen; eine historisch extrem belastete, aber zur Zeit noch wie ein Filter wirkende materielle Einschränkung). Der Sache nach korrigiert der neue $\S 66 \mathrm{~b}$ StGB die Entscheidung des erkennenden Gerichts. Waren die Tatsachen zum Zeitpunkt der Verurteilung also schon bekannt, was schon wegen $\S \S 20,21$ StGB eher die Regel sein wird, müsste schon dies eine Sperrwirkung entfalten ${ }^{7}$, mit der Folge dass das Gesetz nur die Fälle erfassen würde, für die es eigentlich gedacht war, nämlich Altfälle, bei denen die 2002 eingeführte vorbehaltene Sicherungsverwahrung nach $\S 66$ a StGB noch nicht greifen konnte. Nicht verschwiegen werden soll allerdings, dass der Wortlaut der neuen Norm und die von der Ministerin erklärte Absicht weit auseinander klaffen, er verspricht nämlich fast alles, was Bild und andere Massenmedien fordern. Aber der Wunsch nach »Wegschließen « muss vor Gericht durch das Nadelöhr der "neuen Tatsachen « gefädelt werden. Sollten sich aber dennoch diejenigen durchsetzen, die ohnehin fordern, die Vorbehaltslösung des Jahres 2002 abzuschaffen und nur noch über die "nachträgliche Sicherungsverwahrung « zu agieren, dann werden wir demnächst wieder die Karlsruher Richter hören; denn $\$ 66$ b ist weiter gefasst als die $\$ \S 66,66$ a (1998 - 31.03.2004). Eine derart weit gefasste Urteilskorrektur ist ein rechtsstaatlicher Tabubruch, der dem Schweizer Volksbegehren durchaus gleicht und auch hier bislang ohne Vorbild ist. Die Drohung damit allein würde wie ein Damoklesschwert den gesamten Vollzug langer Freiheitsstrafen begleiten $(\S$ 66 b Abs. 2 ermöglicht die Drohung mit einer nachträglichen Sicherungsverwahrung schon bei Ersttätern, wenn sie ursprünglich zu mehr als vier Jahren verurteilt worden waren). Wird die neue Vorschrift schlicht umgesetzt, wird sie die zu mehr als vier Jahren Verurteilten davon abhalten, sich Therapeuten und anderen Ansprechpartnern im Vollzug zu öffnen, zumal der Datenschutz höchst uneinheitlich praktiziert wird, meist aber zumindest eine grobe Einschätzung über den Therapieverlauf seitens der Anstalt möglich ist (und eigentlich ja auch möglich sein sollte). Man braucht den Teufel nicht an die Wand zu malen, um zu sehen, dass symbolische Politik reale Folgen hat.

Störend ist nicht allein der Paradigmenwechsel weg von der Antidiskriminierungspolitik hin zu einer stärkeren Betonung der Sicherheit. Dies war das Erbe der jetzigen Regierung, als sie 1998 angetreten ist. Warum hat man damals diesen Wunsch nach Sicherheit vor einer kleinen Gruppe von Rückfalltätern nicht schlicht akzeptiert und professionell umgesetzt? Vielleicht wäre uns das traurige Schauspiel in mehreren Akten erspart geblieben. Leider blockierte sich aber das Bundesjustizministerium mit der mittlerweile vom BVerfG widerlegten These, eine nachträgliche Sicherungsverwahrung sei Polizeirecht und damit Ländersache. Andere wiederum meinten, die CDU/CSUForderungen als verfassungswidrig abschütteln zu können und wiederholten immer wieder ihre "grundsätzlichen « Bedenken gegen die Sicherungsverwahrung als solche. Politisch führte diese Strategie in die Warteschleife: "wir warten ab, bis uns das BVerfG zwingt", dann können wir ja immer noch nachbessern ${ }^{8}$. Die Zwangslage war leider vorhersehbar! Mehr Sicherheit hätte und könnte noch immer durch eine früher einsetzende durchlässige und flexible Kontrollpolitik erreicht werden. Zurzeit aber sind beide Spuren, die Strafe und die Maßregeln der Besserung und Sicherung zu starr voneinander abgegrenzt, außerdem ist die Behandlung im Maßregelvollzug an zumindest teilweise sachfremde Voraussetzungen gebunden. Bei einer Zuschreibung von Schuldunfähigkeit ist es zwingend und konsequent, dass die Sicherung nur im Psychiatrischen Krankenhaus erfolgen kann. Nicht aber bei der sehr viel größeren Gruppe der vermindert schuldfähigen Täter. Keinen Sinn macht es bei den Fällen einer Dekulpation nach $\S 21$ StGB, die 
zweite Spur für sie zu reservieren, und geradezu widersinnig ist es für chische Störung vorliegt, aber keine Schuldminderung nach § 21, nicht offen zu lassen, wo diese Personen alle Fälle, in denen zwar eine psy- stig nicht einmal die mediale Aufregung. Da sie außerdem noch handfeste negative Folgen hat, verdient als das bezeichnet werden, was sie ist: Kurpfuscherei. sie kein Verständnis, sondern soll

\section{Habituelle Kriminalität wird danach definiert als} eingeschliffenes Verhaltensmuster in der Biografie erkennbar, Delinquenzbeginn in Kindheit oder Jugend, Herkunft aus dissozialem Milieu, gewalttätige Delikte in der Vorgeschichte und gehäuftes Lockerungs- und Bewährungsversagen

\section{(Volker Dittmann, Forensische Abteilung/ psychiatr. Klinik/ Universität Basel).}

am besten behandelt oder gesichert werden können. Erst dann könnte man Zuweisungen zur einen oder zur anderen Einrichtung später korrigieren. Da die Politik zu einer Revision des Sanktionenrechts bislang nicht bereit war, greift man erst ganz am Ende, wenn die Entlassung ansteht, $\mathrm{zu}$ rabiaten Mitteln wie etwa der nachträglichen Sicherungsverwahrung, um Versäumnisse, Fehlentscheidungen und unverscheidbarkeit zu verdecken. Es wäre besser gewesen, die Regierungsparteien hätten das gesamte Reaktionensystem einer Revision unterzogen (und nur eine Übergangsregelung für Altfälle, auf welche die 2002 geschaffene vorbehaltene $\mathrm{Si}$ cherungsverwahrung nicht mehr anwendbar ist, geschaffen), als nun mit unabsehbaren Langzeitwirkungen kurzatmig an Symptomen zu kurieren. Es fehlt also ein Konzept für die große Gruppe der gestörten, aber nicht eindeutig schuldunfähigen bzw. vermindert schuldfähigen Sexualstraftäter. Hier müsste sehr früh erprobt werden, ob, wie und wo behandelt werden kann. Erweist sich, dass Behandlung eher nicht möglich ist, haben wir es mit einem Menschen zu tun, dessen tragisches Schicksal wir bedauern, bei dem aber potentielle Opfer ein Recht darauf haben, dass die staatlichen Einrichtungen professionell reagieren. Symbolische Politik ist das Gegenteil von professioneller Reaktion. Sie ist untaugliche Beruhigungspolitik und lindert langfrimeidliche Situationen der Unent-
Leider verdirbt diese Alibipolitik auch noch den ansonsten sehr guten Eindruck einer insgesamt reflektierten strafrechtlichen Reaktion auf eine in den letzten Jahrzehnten veränderte Sexualmoral. Niemand redet über die gelungene Reform des Sexualstrafrechts, alle reden von der Sicherungsverwahrung. Es drängt sich auch der Eindruck auf, als habe es Gleichstellungspolitik selbst dort schwer, gesehen und gewürdigt zu werden, wo sie überlegt agiert und auch erfolgreich ist. Der Feind des Guten scheint der Glaube an das noch Bessere zu sein. Hoffen wir also auf kluge Richterinnen und Richter, die dieses Gesetz zum Alibigesetz machen und auf eine langfristig angelegte liberale und pragmatische Korrektur der zur Zeit noch offenen Fragen. Sie lauten: wie kann eine professionelle Reaktion, und sei es Behandlung (oder Verwahrung) der Männer organisiert werden, welche die neue Verhandlungsmoral in Fragen einer liberalisierten Sexualität nicht lernen konnten oder es nicht wollen und wie kann die knappe Ressource Therapie und Prognose klug eingesetzt werden.

\section{Fußnoten:}

1 Ich danke den Schweizer Strafrechtslehrern Günter Stratenwerth und Stefan Trechsel für ihre Hilfe beim Verständnis der Schweizer Situation, insbesondere danke ich Stefan Trechsel für seine Informationen zur Frage der EMRK-Konfor- mität der vom erkennenden Gericht nicht vorbehaltenen, sondern echten nachträglichen Sicherungsverwahrung.

2 Dünkel, »Sicherungsverwahrung (erneut) auf dem Prüfstand «, NK 2/04, 42-48.

3 Aus dem amerikanischen Diskurs: Alice Vachss, »Merkt euch ihre Namen!« Eine Staatsanwältin im Kampf gegen Vergewaltiger, Pädophile und ihre Lobby,1993; Anita Heiliger, Aktiv gegen Männergewalt, Frauenoffensive, 1998; dies., Sexuelle Gewalt. Männliche Sozialisation und potentielle Täterschaft, 1995; im strafrechtswissenschaftlichen Schrifttum immerhin eine Dissertation, die sich weitgehend der Empörungsliteratur verschreibt: Susanne Folkers, Ausgewählte Probleme bei sexueller Nötigung und Vergewaltigung aus der Sicht der Praxis,2004.

4 Vgl. hierzu Günter Hinrichs, Neue Ansätze oder »alter Wein in neuen Schläuchen «, NK 3/2003, 89-91.

5 Am 18.06.2004 beschloss der Bundestag den mittlerweile in Kraft getretenen modifizierten Regierungsentwurf BT-Drucks. 15/2887 i.d.F. von BT-Drucks. 15/2945. Der Bundesrat versucht in seiner Empfehlung die nachträgliche Sicherungsverwahrung zum Regelfall zu machen, aber auch die Bundesregierung lässt nicht erkennen, dass sie ein Konzept hat. Sie ist schlicht unter Druck gesetzt worden, lässt aber keinen Lernprozess erkennen. Statt dessen wird ein Loch nach dem anderen gestopft, aber nicht gesehen, dass der konzeptionslose Aktionismus des Jahres 1998 seitens der Vorgänger dieser Regierung kein sinnvolles Sicherheitskonzept hinterlassen hat, sondern eine verhängnisvoll wirkende Alibi-Gesetzgebung, die - wird der Denkfehler nicht eingesehen - noch weitere Alibi-Gesetze schaffen wird.

6 Günter Stratenwerth, Zur Rolle der sog. "Fachkommissionen «, in: FS für Stefan Trechsel zum 65.Geburtstag, hrsg. von Donatsch/Forster/ Schwarzenegger, 2002; ferner Andrea Baechthold, Die Fachkommissionen zur beurteilung der Gemeingefährlichkeit - cui bono? In:

Bauhofer/Bolle/Dittmann (Hrsg.), Gemeinefährliche« Straftäter, 200, $325 \mathrm{ff}$.

7 Braum ZRP 4/2004, 105 (107)

8 Die Fachzeitschriften sind voll mit Anregungen, vgl. Callies, ZfStrVollz und Straffälligenhilfe 3/2004, $135 \mathrm{ff}$.

6 Die Fachzeitschriften sind voll mit Anregungen, vgl. Callies, ZfStrVollz und Straffälligenhilfe 3/2004, $135 \mathrm{ff}$.

\section{TERMINAL}

\section{Die rechtliche Bewältigung von Erscheinungsformen organisierter Kriminalität}

von Jörg Kinzig,

Band 17, Schriftenreihe Strafrecht und Kriminologie, Untersuchungen und Forschungsberichte aus dem Max-Planck-Institut f. ausl. u. intern. Strafrecht Freiburg i. Br., Duncker \& Humblot Berlin, 2004, S. 849, 98,- €, Habilitationsschrift

Kinzig selbst beschreibt sein Ziel als kritische Bestandsaufnahme der Sachverhalte, welche von Polizei und Justiz unter dem Begriff OK aufgegriffen und Beschreibung, wie und mit welchem Ergebnis sie von der Justiz bewältigt werden. Die Schwerpunkte liegen auf drei Aspekten:

- Begriffsgeschichte

- strafprozessrechtliche und kriminalpolitische Klärung der Folgen der intensiveren Eingriffsbefugnisse bei einer Strafverfolgung in sog. OK - Fällen

- Hellfeldstudie durch vergleichende Aktenanalyse und Interviews mit Betroffenen

Zum Abschluss wird der Frage nachgegangen, ob sich aus der Untersuchung die Tragfähigkeit der bisherigen Definitionsversuche ergibt bzw. weitergehend, ob sich eine Form kriminellen Verhaltens feststellen lässt, die sinnvollerweise mit dem Begriff OK belegt werden kann. 